\title{
OA07.01. A naturopathic approach to the prevention of cardiovascular disease: a cost effectiveness analysis of a randomized multi- worksite trial
}

\author{
P Herman ${ }^{1 *}$, O Szczurko ${ }^{2}$, K Cooley $^{2}$, D Seely ${ }^{3}$ \\ From International Research Congress on Integrative Medicine and Health 2012 \\ Portland, Oregon, USA. 15-18 May 2012
}

\section{Purpose}

Cardiovascular disease (CVD), a major cause of death and substantial healthcare costs, is also largely preventable. The purpose of this study is to determine the costeffectiveness of a naturopathic approach to the primary prevention of cardiovascular disease.

\section{Methods}

This study is an economic evaluation alongside a pragmatic, multi-worksite, randomized trial comparing enhanced usual care (EUC; usual care plus biometric screening) to the addition of a naturopathic approach to CVD prevention (NC+EUC; an individualized package of lifestyle counseling and nutritional medicine). Biometric and self-report outcomes were collected at 0,6 , and 12 months. Cost-effectiveness is determined from the societal and employer perspectives for participants who consented (before randomization) to make available their electronic employer sick leave and medical claims data.

\section{Results}

Of the 246 employees who consented to the trial, twothirds also gave consent to make available their claims and sick leave data: 77 (63.1\% of 122) randomized to EUC and 79 (63.7\% of 124) randomized to NC+EUC. There were no statistically significant differences between those who did and did not give this consent across baseline characteristics, outcomes, or tendency to miss study visits. After one year $\mathrm{NC}+\mathrm{EUC}$ resulted in a net decrease of 3.3 (CI: $1.7-4.8$ ) percentage points in 10-year CVD event risk (NNT=30) and 0.9 (CI: $0.2-$ 1.6) points in CVD mortality risk $(\mathrm{NNT}=111)$. These risk reductions came with an average net savings of $\$ 1138$ in societal costs and $\$ 1187$ in employer costs. There was no change in quality-adjusted life-years across the study year.

\section{Conclusion}

In this multi-worksite-based study, a naturopathic approach to the primary prevention of CVD provided significant reductions in CVD risk over usual care plus biometric screening. These risk reductions are achieved with cost savings to society and employers. Further research into naturopathic approaches to CVD risk reduction is justified.

\section{Author details}

${ }^{1}$ University of Arizona, Tucson, USA. ${ }^{2}$ Canadian College of Naturopathic Medicine and University of Toronto, Toronto, Canada. ${ }^{3}$ Canadian College of Naturopathic Medicine, Toronto, Canada.

Published: 12 June 2012

\section{doi:10.1186/1472-6882-12-S1-O25}

Cite this article as: Herman et al:: OA07.01. A naturopathic approach to the prevention of cardiovascular disease: a cost effectiveness analysis of a randomized multi-worksite trial. BMC Complementary and Alternative Medicine 2012 12(Suppl 1):O25.

${ }^{1}$ University of Arizona, Tucson, USA

Full list of author information is available at the end of the article

(c) 2012 Herman et al; licensee BioMed Central Ltd. This is an Open Access article distributed under the terms of the Creative Commons Attribution License (http://creativecommons.org/licenses/by/2.0), which permits unrestricted use, distribution, and reproduction in any medium, provided the original work is properly cited. 